\title{
INTERLOCKING NAILING OF HUMERAL SHAFT FRACTURES A RETROSPECTIVE STUDY OF 114 PATIENTS
}

\author{
DEMIREL M, TURHAN E, DEREBOY F, OZTURK A
}

\section{ABSTRACT}

\begin{abstract}
BACKGROUND: Fractures of the humeral shaft are relatively common injuries. Literature suggests that humeral shaft fractures represent approximately $3 \%$ of all fractures. There are several modalities for the management of diaphyseal humeral fractures. The latest investigations emphasize the concept of minimal exposure and rigid fixation. AIM: The aim of the study is to evaluate the results of antegrade intramedullary nailing in humeral shaft fractures. DESIGN: A retrospective review SETTINGS: Patients were treated in private hospital settings by 3 orthopaedics surgeon Material and Methods: Between 1995 and 2003, the technique of antegrade locked intramedullary nailing with UHN in humeral shaft fractures was performed on 114 patients. Forty-two (36\%) patients sustained multiple traumas, and 22 (19\%) fractures were open. The outcomes were evaluated with a mean follow-up of 41 months. STATISTICAL ANALYSIS USED: Ranges of results given RESULTS: In 109 fractures primary union observed. In the other five patients union achieved after removal of the nail and fixation with DCP and bone grafting. The average time for union was 13 weeks (range, 10-36 weeks). One hundred-five patients had excellent or satisfactory recovery of shoulder and elbow function. Complications included impingement due to proximal locking screws in two patients and prominent nail in three patients, transient postoperative radial nerve palsy in four patients. CONCLUSIONS: This study shows that antegrade locked nailing in humeral shaft fractures are reliable and also effective in multiply injured patients.
\end{abstract}

Key Words: Humerus fracture, Intramedullary nail, shoulder problems, plate fixation

\section{INTRODUCTION}

There are several modalities for the management of diaphyseal humeral

Departments and institutions, Ankara Bayindir Hospital Orthopaedics\&Traumatology

Correspondence

Murat Demirel

Turan Gunes Bulv 71. sok 14/5 Cankaya 06550 Yildiz

Ankara Turkey, E-mail: drmdemirel@gmail.com fractures. ${ }^{[1,2]}$ Most acute humeral diaphyseal fractures can be treated adequately using nonoperative methods. With improved implant design and surgical technique, operative treatment of humeral diaphyseal fractures increasingly has become accepted. ${ }^{[1]}$ Indications for surgical treatment of humeral diaphyseal fractures are open fractures, segmental fractures, bilateral fractures, floating elbow injuries, fractures associated with 
vascular injuries or progressive neurological injury, and fractures in patients with multiple injuries. ${ }^{[1,2]}$ Such treatment offers several advantages. Although plate osteosynthesis can afford a rigid fixation and good functional recovery, its disadvantages have been reported like plate fixation does require a wide surgical exposure and more time when compared with intramedullary fixation. ${ }^{[1,2,3,4]}$ Locked intramedullary nails usually can be inserted using closed techniques, avoiding the extensive soft tissue dissection required for plating. Interlocking nails gives rotational stability, decrease the need for postoperative bracing and allowing early mobilization of the extremity. ${ }^{[3,4]}$

In this article we present our experience with locked antegrade nailing of acute humeral diaphyseal fractures in 114 patients.

\section{MATERIALS AND METHODS}

This is a retrospective review of 114 acute humeral diaphyseal fractures in 114 patients aged between 17- 88 years (average, 44.5 years), treated with antegrade $A O$ unreamed humeral nail (AO-UHN, Synthes, Paoli, PA) for acute humeral fractures $(n=108)$ or for metastatic bone invasion at our hospital between January 1995 and December 2003 [Table 1]. Information was obtained through

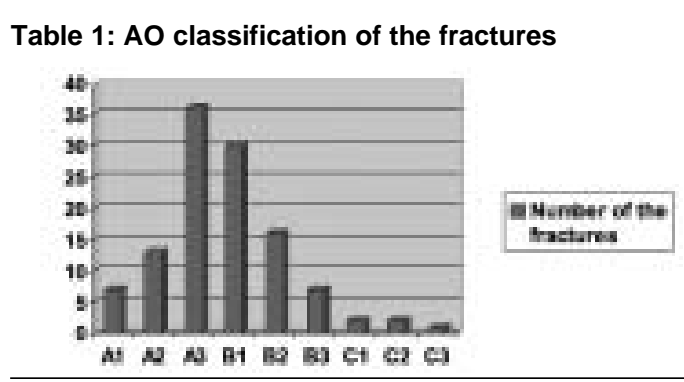

review of clinic notes, photographs, therapy measurements and operative notes. All aspects of this study were approved by the Hospital's Review Board. The exclusion criteria of this study were fractures involving adjacent joints, bone defect $>3 \mathrm{~cm}$, infection at the fracture site and patients younger than 17 years old.

There were 79 male and 35 female patients. Mechanisms of injury included 85 motor vehicle accidents, two gunshot wounds, twelve pedestrian versus motor vehicle accidents, eleven falls from a height, four motorcycle accidents. Associated injuries occurred in 42 acute fractures: five head injury, eight chest injury, nine abdominal injuries, and twenty multiple fracture. Pathologic fractures were caused by metastasis from one breast cancer, two prostate cancers, one lung cancer, and two multiple myeloma. The average delay from trauma to operation was 12 hours (range, 2 hours- 6 days; median, 18 hours). No bone grafting or bone cementing was performed in this study. There were twelve primary nerve palsies. For acute fractures, static locking was performed in 99 cases and dynamic locking in fifteen. Static locking was performed in all six pathologic fractures.

\section{Technique}

Fixation with AO-UHN is appropriate for humeral fractures between $3 \mathrm{~cm}$ proximal to the olecranon fossa and $2 \mathrm{~cm}$ distal to the surgical neck. All nails in this series were placed in an antegrade fashion and $86,8 \%$ of them were statically locked. Patients are positioned supine on a radiolucent operating table. The ipsilateral shoulder is pulled to the edge of the table, and the head is turned to face the contralateral side. The fluoroscopic imager is placed perpendicular to the operating table, ipsilateral to the injured extremity to allow easy anteroposterior imaging. A scapular $Y$ view of the shoulder is obtained by rotating the $\mathrm{C}$ arm approximately $30^{\circ}$ to $45^{\circ}$.

All open fractures are treated with immediate debridement and irrigation and exploration of the fracture site to ensure that the radial nerve is not entrapped. The entry portal for the nail is made using the anterolateral approach described by Riemer et al. ${ }^{[5]}$ A $2-\mathrm{cm}$ incision is made anterior to the midpoint of the acromion and the deltoid muscle is split longitudinally. A $1-\mathrm{cm}$ incision is made in the rotator cuff in line with its fibers. An awl is used to create the entry postal just medial to the greater tuberosity in the sulcus between the greater tuberosity and the articular margin. The nails inserted without reaming. Canal diameter was measured on preoperative radiographs. The nail is inserted until its tip lies 1.5 to $2 \mathrm{~cm}$ proximal to the olecranon fossa. The proximal end of the nail is seated approximately $5 \mathrm{~mm}$ beneath the bone to prevent impingement. The nail should be inserted with the fracture well aligned to avoid intraoperative comminution. Proximal interlocking screw is placed with the use of a proximal drill guide. The screw should be directed so that it exits medially, distal to the articular margin of the humerus. A drill sleeve is inserted through a stab incision after soft tissues have been dissected bluntly down to bone. A hole is made with a $2.7-\mathrm{mm}$ drill bit, and a 4-mm bicortical screw is inserted. Distal locking was performed using a freehand technique. Before the distal screw was inserted, the fracture site is compressed by placing an axial load on the elbow. Correct rotation is obtained by pointing the forearm and hand perpendicular to the ceiling.
The patient's arm was supported simply in a neck sling for the first few days after surgery. Range of motion (ROM) exercise was encouraged as early as tolerable., For acute fractures, the patients and radiographs were examined every 2 or 3 weeks until union was achieved. The operation time, time to union, shoulder functional score based on American Shoulder and Elbow Surgeons' score, the visual analogue pain score were recorded. The mean follow-up time was 41 months (range, 18-68 months).

\section{Statistical Analysis}

Mean values of operation time, time to union, shoulder functional score and visual analogue pain scores given with their own ranges.

\section{RESULTS}

Two cases X-rays, managed with intramedullary nailing presented in [Figure $1 \mathrm{~A}$ $B]$ and [Figure 2A-B].One hundred-nine of the $114(95.6 \%)$ fractures eventually united. The average time to union was 13 weeks (range, 10-36 weeks). Five fractures required autogenous bone grafting for delayed union or nonunion. Three of these five were either open fractures (Type II or Type IIIC) or closed fractures treated with an open technique. All fractures united after removal of the nail and fixation with DCP and bone grafting.

To assess function, we used the American Shoulder and Elbow Surgeons' (ASES) shoulder score for 13 activities of daily living requiring full shoulder and elbow movement [Table 2]. The maximum possible score is 52 points. The average score was 48,5 (range, 40-52). We quantified pain using visual 
Table 2: Details of the American Shoulder and Elbow Surgeons' (ASES) score ( $4=$ normal $; 3=$ mild compromise ; 2 = difficulty ; $1=$ with aid ;0 = unable ; NA = not available) Back pocket
Wash opposite Wash opposite axilla
Comb hair Carry 101b at side Sleep on affected side Perineal care
Eat with utensil Use arm at shoulder leve Dress Pull

analogue scales, with zero being no pain and 10 extreme pain. The results range between zero and two with average 1,5. Complications included impingement due to proximal locking screws in two patients and prominent nail in three patients, transient postoperative radial nerve palsy in four patients.

For fractures that were nailed in a closed manner, the operation time averaged 50 minutes (range, 38-122 minutes). For the twelve fractures treated with open nailing, the average operation time was 115 minutes.

\section{DISCUSSION}

Isolated, low energy humeral shaft fractures

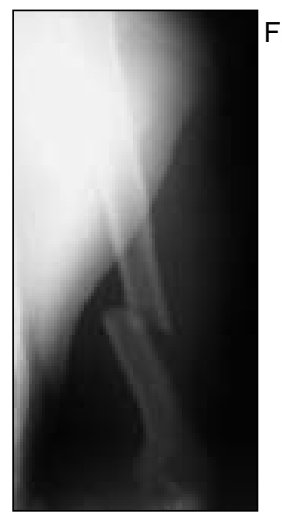

\section{Fig1}

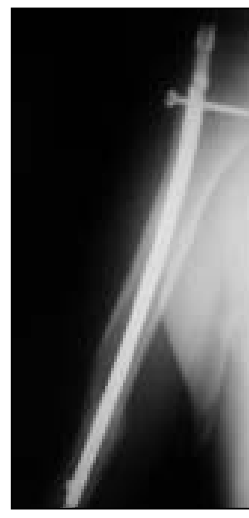

Figure 1: Preoperative $\mathrm{X}$-ray of humeral shaft fracture. Figure 2: After union achieved. usually can be treated satisfactorily with nonoperative methods, ${ }^{[7,8]}$ but operative stabilization often is necessary for acute, high energy humeral shaft fractures to improve healing, fracture alignment, and functional results. ${ }^{[9,10]}$ In previous studies, the union rate of antegrade nailing of acute humeral fractures using nails with transfixing screws has ranged from $71 \%$ to $100 \%{ }^{[3,11,12,13]}$ However, the current authors also think that some lower union rates among reported clinical results might be accounted for by other factors, such as improper nailing direction, over-distraction of the fractures, or insufficient fixation stability, all of which could impair healing.

When we look at the union rates, the reported recovery of shoulder function after antegrade nailing varies. In studies using Russell-Taylor nails, Ikpeme reported 22 (88\%) patients with excellent or satisfactory recovery of shoulder function according to their Neer score. ${ }^{[12]}$ Postoperative shoulder pain was caused mostly by proximal locking screws. Crates and Whittle reported 66 of 73 (90\%) patients had full recovery of shoulder function. ${ }^{[3]}$ Three

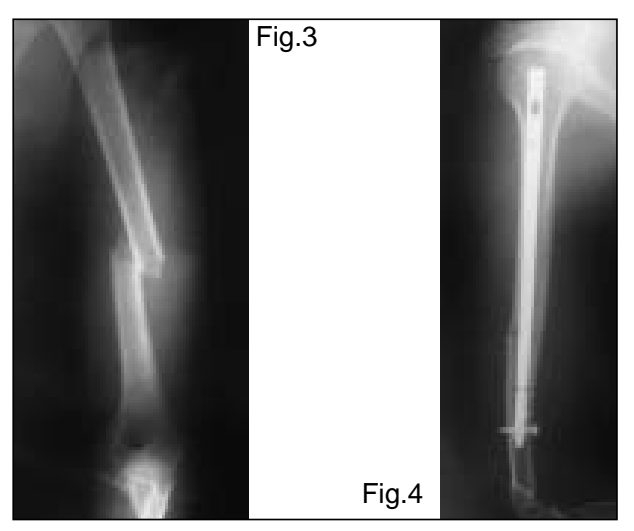

Figure 3: Preoperative X-ray of humeral shaft fracture. Figure 4: After union achieved patients had impingement: two caused by the proximal locking screw and one by a prominent nail. In contrast, Hems and Bhullar reported only 11 of $15(73.3 \%)$ patients had excellent or satisfactory Neer scores. ${ }^{[11]}$ They attributed these less than satisfactory results to intraoperative rotator cuff injury. However, their follow-up time (range, 1-42 months) was insufficient in terms of recovery of shoulder function. In the current study, with the exception of three $(7.9 \%)$ patients with severe associated injury or bad general condition, patients with acute fractures had excellent or satisfactory recovery of shoulder joint function. The current authors think the following technical factors might be responsible for poor recovery of shoulder function: massive injury of the rotator cuff with inadequate repair, prominent nail head or locking screws, axillary nerve injury, and intra-operative comminution of the humeral head.

More rigid locked intramedullary nails have better rotational control than flexible nails, which theoretically should decrease the frequency of nonunion. Riemer et al reported no nonunions in 28 acute humeral shaft fractures treated with Seidel nails. ${ }^{[5]}$ Robinson et al, however, evaluated 30 humeral fractures treated with Seidel nails and found that $23 \%$ required additional treatment for delayed union.[15] Ingman and Waters reported union in $95 \%$ of 21 humeral fractures treated with modified Grosse-Kempf nails. ${ }^{[13]}$ Ikpeme reported no nonunions in 30 acute humeral fractures treated with Russell-Taylor nails. ${ }^{[12]}$ Lin et al, reported union in all 47 fractures treated with locked nailing. ${ }^{[2]}$ Crates et al reported $94.5 \%$ union primarily with antegrade Russell-Taylor humeral nailing and two additional fractures united after bone grafting. ${ }^{[3]}$

The most frequent criticism of antegrade humeral nailing has been its potentially deleterious effect on shoulder function. In a series of humeral fractures stabilized predominantly by Rush rods, Stern et al reported the development of adhesive capsulitis in $56 \%$ of fractures treated with antegrade nailing. ${ }^{[15,16]}$ The insertion point violated the rotator cuff in most of these patients, and nails frequently migrated proximally. Shoulder function returned to near normal after hardware removal. In 28 humeral fractures stabilized with antegrade Rush or Ender nails, Brumback et al reported excellent results in $18(64 \%)$, good results in seven $(25 \%)$, and poor results in three (11\%). ${ }^{[9]}$ Eight of the 10 shoulders with good or poor function had nails inserted through the rotator cuff. Seven of the eight had impingement symptoms develop that required hardware removal. Stern et al Brumback et al recommended an antegrade insertion point lateral and distal to the rotator cuff. ${ }^{[9,16]}$ Shoulder problems also have been reported with antegrade insertion of more rigid nails. Robinson et al reported that 12 of $30(40 \%)$ humeral fractures treated with Seidel nails had protrusion of the nail above the humeral tuberosity, usually because of failure of the locking mechanism. ${ }^{[15]}$ Five other patients in whom the nail was prominent also had poor shoulder function, which the authors attributed to local rotator cuff damage during insertion. Riemer et al also reported that five of 12 patients in whom a Seidel nail was inserted through a lateral deltoid incision had persistent shoulder stiffness. ${ }^{[5]}$ No patients with nails inserted through an anterior deltoid 
incision had restricted shoulder motion; however, it sometimes took as many as 6 months for full shoulder function to return.

In a series of 30 acute humeral fractures treated with antegrade Russell-Taylor nails, Ikpeme reported that six (20\%) patients had shoulder pain and decreased shoulder abduction because of impingement of the proximal locking screws. ${ }^{[12]}$ Removal of the proximal screws in five of these patients resulted in complete resolution of symptoms. Two other patients had proximal nail migration because of proximal interlocking through an area of comminution.

In our series, shoulder pain because of nail impingement occurred in five (4.3\%) patient in whom the nail was not properly countersunk. Shoulder pain was related to the proximal locking screws in two (1.7\%) patients. Symptoms resolved in all patients after hardware removal.

This retrospective review of 114 humeral shaft fractures treated with AO-UHN system revealed acceptable data's in time to union, shoulder and elbow function, the operation time. Antegrade interlocking humeral nailing does not require extensive soft tissue dissection, infrequently requires bone grafting, does not require external immobilization, and may be more suitable for comminuted and segmental fracture patterns than plating or flexible nailing techniques. We believe that antegrade locked nailing in humeral shaft fractures are reliable and also effective in multiply injured patients.

\section{REFERENCES}

1. Sarmiento A, Waddellle JP, Latta LL. Diaphysea humeral fractures: treatment options. Inst Course Lect 2002;51:257-69.

2. Lin J, Hou SM. Antegrade locked nailing for humeral shaft fractures. Clin Orthop Relat Res 1999;365:201-10.

3. Crates J, Whittle AP. Antegrade interlocking nailing of acute humeral shaft fractures. Clin Orthop Relat Res 1998;350:40-50.

4. Scheerlinck T, Handelberg F. Functional outcome after intramedullary nailing of humeral shaft fractures: Comparison between retrograde Marchetti-Vicenzi and unreamed AO antegrade nailing. J Trauma 2002;52:60-71.

5. Riemer BL, Butterfield SL, D'Ambrosia R, Kellam $J$. Seidel intramedullary nailing of humeral diaphyseal fractures: A preliminary report. Orthopedics 1991;14;239-46.

6. Rockwood C, Matsen F. The Shoulder. WB Saunders: Philadelphia; 1990. p. 161

7. Balfour GW, Mooney V, Ashby ME. Diaphyseal fractures of the humerus treated with a readymade fracture brace. J Bone Joint Surg 1982;64A:11-3.

8. Sarmiento A, Kinman PB, Galvin EG, Schmit $\mathrm{RH}$, Phillips JG. Functional bracing of fractures of the shaft of the humerus. J Bone Joint Surg 1977;59A:596-601.

9. Brumback RJ, Bosse MJ, Poka A, Burgess AR Intramedullary stabilization of the humeral shaft fractures in patients with multiple trauma. J Bone Joint Surg Am 1986:68:960-70

10. Henley MB, Chapman JR, Claudi BF. Closed retrograde Hackethal nail stabilization of humeral shaft fractures. J Orthop Trauma 1992;6:18-24.

11. Hems TE, Bhullar TP. Interlocking nailing of humeral shaft fractures: The Oxford experience 1991 to 1994 . Injury 1996;27:485-9

12. Ikpeme JO. Intramedullary interlocking nailing for humeral fractures: Experiences with the RussellTaylor humeral nail. Injury 1994;25:447-55.

13. Ingman AM, Waters DA. Locked intramedullary nailing of humeral shaft fractures. $J$ Bone Joint Surg 1994;76B:23-9.

14. Lin J. Treatment of humeral shaft fractures with humeral locked nail and comparison with plate fixation. J Trauma 1998;44:859-64.
15. Robinson CM, Bell KM, Court-Brown CM McQueen MM. Locked nailing of humeral shaft fractures. Experience in Edinburgh over a twoyear period. J Bone Joint Surg Br 1992;74:55862.

16. Stern PJ, Mattingly DA, Pomeroy DL, Zenni EJ Jr, Kreig JK. Intramedullary fixation of humeral shaft fractures. J Bone Joint Surg 1984;66A:639-46.
Indian Journal of Medical Sciences is pleased to announce the launch of its website. The URL of the website is http://www.indianjmedsci.org.

The features of the site are:

- Free full text availability of articles in HTML as well as PDF

- Link to abstracts and full text from the cited references

- Link to PubMed abstracts of published articles by authors

- Link to related articles in PubMed

- Link from text of articles to various databases and search engines

- Facility to submit comments on articles

- Email notifications on new issue release

- Statistics of articles download and visits

- Structure based on OpenURL, DC Metadata and other international standards 